\title{
On the Cauchy Problem for a Nonlinearly Dispersive Wave Equation
}

\author{
Zhaoyang YIN \\ Permanent address: Department of Mathematics, \\ Zhongshan University, 510275 Guangzhou, China \\ E-mail: mcsyzy@zsu.edu.cn \\ Present address: Department of Mathematics, Lund University, \\ P.O. Box 118, SE-221 00 Lund, Sweden \\ E-mail: yin@maths.lth.se \\ Received May 15, 2002; Accepted August 19, 2002
}

\begin{abstract}
We establish the local well-posedness for a new nonlinearly dispersive wave equation and we show that the equation has solutions that exist for indefinite times as well as solutions which blowup in finite time. Furthermore, we derive an explosion criterion for the equation and we give a sharp estimate from below for the existence time of solutions with smooth initial data.
\end{abstract}

\section{Introduction}

In this letter we consider the Cauchy problem for the nonlinear equation:

$$
\begin{aligned}
& u_{t}-u_{t x x}+2 \omega u_{x}+3 u u_{x}=\gamma\left(2 u_{x} u_{x x}+u u_{x x x}\right), \quad t>0, \quad x \in \mathbb{R}, \\
& u(0, x)=u_{0}(x), \quad x \in \mathbb{R},
\end{aligned}
$$

with $\omega \geq 0$ and $\gamma \in \mathbb{R}$ fixed constants.

With $\gamma=1$ in equation (1.1) we find the Camassa-Holm equation for the unidirectional propagation of shallow water waves over a flat bottom, $u(t, x)$ standing for the fluid velocity at time $t \geq 0$ in the spatial $x$ direction and $\omega$ being a nonnegative parameter related to the critical shallow water speed (see [3] and [16]). In this case equation (1.1) has a biHamiltonian structure 14 and is completely integrable (see 1, 6, 18]). The solitary waves are smooth if $\omega>0$, they become peaked in the limiting case $\omega=0$, and interact like solitons [3].

For $\gamma=0$, equation (1.1) becomes the regularized long wave equation, a well-known equation for surface waves in a channel 2]. All solutions are global and the solitary waves are smooth. Despite having a Hamiltonian structure, the equation is not integrable and its solitary waves are not solitons [13]. 
For $\omega=0$ and $\gamma \in \mathbb{R}$, equation (1.1) particularizes to a nonlinear dispersive model for finite-length and small-amplitude radial deformation waves in thin cylindrical compressible hyperelastic rods (see [10] and [1] ), $u(t, x)$ representing the radial stretch relative to a prestressed state. The case $\gamma=1$ (limiting case of the Camassa-Holm equation) has been extensively studied: the equation has global solutions [5, 7] and also solutions which blowup in finite time [5, 8, 7. It is an integrable Hamiltonian system [1, 6] and its solitary waves are peaked and interact like solitons 3 .

For $\omega>0$ and $\gamma \in \mathbb{R}$, equation (1.1) seems not yet to have been discussed. The aim of this letter is to prove local well-posedness of strong solutions to equation (1.1) for a large class of initial data, to analyze global existence of smooth solutions, as well as to get a blow-up criterion and a sharp estimate from below of the existence time for strong solutions.

\section{Local Well-posedness}

To prove the local existence of solutions for equation (1.1) we will apply Kato's semigroup approach [17]. For this purpose, let us reformulate equation (1.1) as a quasi-linear evolution equation for $y:=u-u_{x x}$,

$$
y_{t}+\gamma y_{x} u+2 \gamma y u_{x}+2 \omega u_{x}+3(1-\gamma) u u_{x}=0, \quad t>0, \quad x \in \mathbb{R} .
$$

Note that if $p(x):=\frac{1}{2} e^{-|x|}, x \in \mathbb{R}$, then $\left(1-\partial_{x}^{2}\right) f=p * f$ for all $f \in L^{2}(\mathbb{R})$ so $p * y=u$, where $*$ denotes convolution. Using this identity, we can rewrite equation (2.1) as follows,

$$
u_{t}+\gamma u u_{x}+\partial_{x} p *\left(\frac{3-\gamma}{2} u^{2}+\frac{\gamma}{2} u_{x}^{2}+2 \omega u\right)=0, \quad t>0, \quad x \in \mathbb{R} .
$$

Equation (2.2) is suitable for applying Kato's theory. A framework that is similar to those provided in [7] and [20] can be constructed. As an outcome, we obtain

Theorem 1. Given $u_{0} \in H^{s}(\mathbb{R}), s>\frac{3}{2}$, there exists a maximal $T=T\left(\gamma, \omega, u_{0}\right)>0$, and a unique solution $u$ to equation (1.1), such that

$$
u=u\left(\cdot, u_{0}\right) \in C\left([0, T) ; H^{s}(\mathbb{R})\right) \cap C^{1}\left([0, T) ; H^{s-1}(\mathbb{R})\right) .
$$

Moreover, the solution depends continuously on the initial data.

Theorem 1 contains as particular cases (for various choices of the constants $\gamma$ and $\omega$ ) the well-posedness results obtained in [7, 15, 19] and [20].

\section{Global solutions}

To answer affirmatively the question whether there are solutions of equation (1.1) that exist indefinitely in time, we prove that for every choice of $\gamma \in \mathbb{R}$ and $\omega>0$ there are smooth solitary wave solutions $u(t, x)=\varphi_{c}(x-c t)$ propagating at certain speeds $c>0$.

If $u(t, x)=\varphi_{c}(x-c t)$ is a smooth solitary wave solution of equation (1.1), then

$$
-c \varphi_{c}^{\prime}+c \varphi_{c}^{\prime \prime \prime}+3 \varphi_{c}^{\prime} \varphi_{c}+2 \omega \varphi_{c}^{\prime}=2 \gamma \varphi_{c}^{\prime} \varphi_{c}^{\prime \prime}+\gamma \varphi_{c} \varphi_{c}^{\prime \prime \prime} .
$$


Fix $x \in \mathbb{R}$ and integrate equation $(3.1)$ on $(-\infty, x]$ to obtain

$$
(2 \omega-c) \varphi_{c}+c \varphi_{c}^{\prime \prime}+\frac{3}{2}\left(\varphi_{c}\right)^{2}=\frac{\gamma}{2}\left(\varphi_{c}^{\prime}\right)^{2}+\gamma \varphi_{c} \varphi_{c}^{\prime \prime} .
$$

Multiplying equation $(3.2)$ by $\varphi_{c}^{\prime}$, an integration on $(-\infty, x]$ leads to

$$
\left(\varphi_{c}^{\prime}\right)^{2}\left(c-\gamma \varphi_{c}\right)=\varphi_{c}^{2}\left(c-2 \omega-\varphi_{c}\right) .
$$

A detailed analysis of the above ordinary differential equation yields the following result.

Theorem 2. Let $\omega>0$ and $\gamma \in \mathbb{R}$. Then for every $c>0$ satisfying $c(\gamma-1)<2 \omega \gamma$ and $c>2 \omega$, there exists a nontrivial smooth solitary wave solution $u(t, x)=\varphi_{c}(x-c t)$ of equation (1.1) and its profile $\varphi_{c}$ decays exponentially to zero at infinity.

\section{Solutions with finite life-span}

In this section we address the question of the formation of singularities for solutions to equation (1.1). It turns out that there are smooth initial data for which the corresponding solution to equation (1.1) does not exist globally in time. The precise blowup scenario is described. We also give an explosion criterion and a sharp estimate from below for the maximal existence time of smooth solutions to equation (1.1). Analogous to the case of the Camassa-Holm equation [4], equation (1.1) can be written in Hamiltonian form and has the invariant

$$
E(u)=\int_{\mathbb{R}}\left(u^{2}+u_{x}^{2}\right) d x .
$$

The invariance of $E(u)$ ensures that all solutions of equation (1.1) are uniformly bounded as long as they exist. Arguments similar to those developed in [9] for the elasticity model can be pursued to obtain the following result.

Theorem 3. Given $u_{0} \in H^{s}(\mathbb{R}), s>\frac{3}{2}$, the solution $u=u\left(\cdot, u_{0}\right)$ of equation (1.1) is uniformly bounded. Blow up in finite time $T<+\infty$ occurs if and only if

$$
\liminf _{t \uparrow T}\left\{\inf _{x \in \mathbb{R}}\left[\gamma u_{x}(t, x)\right]\right\}=-\infty .
$$

As an interesting outcome of the previous result, note that in the special case $\gamma=0$, all solutions to equation (1.1) are global. Also, in the particular cases when equation (1.1) models water waves or waves on an elastic rod, the previously described blowup corresponds physically to a breaking wave, respectively rod.

We discuss now the question of finite time blow-up of solution to equation (1.1) with rather general initial data. Let $T>0$ be the maximal existence time of the solution $u(t, \cdot)$ of equation (1.1) with initial data $u_{0} \in H^{s}(\mathbb{R}), s>\frac{3}{2}$. Differentiate equation (2.2) with respect to $x$, and multiply the obtained equation by $\gamma$. Since $\partial_{x}^{2} p * f=p * f-f$, we have

$$
\begin{aligned}
\gamma u_{t x}= & -\frac{\gamma^{2}}{2} u_{x}^{2}-\gamma^{2} u u_{x x}+\frac{(3-\gamma) \gamma}{2} u^{2} \\
& +2 \omega \gamma u-p *\left(\frac{(3-\gamma) \gamma}{2} u^{2}+\frac{\gamma^{2}}{2} u_{x}^{2}+2 \omega \gamma u\right) .
\end{aligned}
$$


Define $m(t)=\gamma u_{x}(t, \xi(t))=\inf _{x \in \mathbb{R}}\left\{\gamma u_{x}(t, x)\right\}$, where $\xi(t)$ is any point where this minimum is attained. Since we deal with a minimum, $u_{x x}(t, \xi(t))=0$ for all $t \in[0, T)$. On the other hand, the regularity of the solution $u(t, x)$ provided by Theorem 1 allows us to infer from the main result in $[8]$ that the function $m(t)$ is almost everywhere differentiable on $(0, T)$, with

$$
\frac{d m}{d t}=\gamma u_{t x}(t, \xi(t)) \quad \text { a.e. } \quad \text { on }(0, T) .
$$

Hence we obtain that

$$
\begin{aligned}
m^{\prime}(t)= & -\frac{1}{2} m^{2}(t)+\frac{(3-\gamma) \gamma}{2} u^{2}(t, \xi(t))+2 \omega \gamma u(t, \xi(t)) \\
& -p *\left(\frac{(3-\gamma) \gamma}{2} u^{2}+\frac{\gamma^{2}}{2} u_{x}^{2}+2 \omega \gamma u\right)(t, \xi(t)) .
\end{aligned}
$$

Involved manipulations of the equations (4.2) and (4.3) and estimates analogous to those in 12 for the special case of the Camassa-Holm equation lead us to the following result.

Theorem 4. (i) Given $u_{0} \in H^{s}(\mathbb{R}), s>\frac{3}{2}$, and assume that we can find $x_{0} \in \mathbb{R}$ with

$$
\gamma u_{0}^{\prime}\left(x_{0}\right)<-\left(\frac{|(\gamma-3) \gamma|}{2} E\left(u_{0}\right)+4 \sqrt{2} \omega|\gamma|\left(E\left(u_{0}\right)\right)^{1 / 2}\right)^{1 / 2}, \quad \gamma \neq 0
$$

Then the corresponding solution to equation (1.1) blows up in finite time.

(ii) Given $u_{0} \in H^{s}(\mathbb{R}), s>\frac{3}{2}$, the maximal existence time of the solution to equation (1.1) with initial data $u_{0}$ is estimated from below by

$$
T_{u_{0}}=\left\{\begin{array}{l}
\frac{-2 \arctan \frac{\left(\frac{(3-\gamma) \gamma}{2} E\left(u_{0}\right)+4 \sqrt{2} \omega|\gamma|\left(E\left(u_{0}\right)\right)^{1 / 2}\right)^{1 / 2}}{m(0)},}{\left(\frac{(3-\gamma) \gamma}{2} E\left(u_{0}\right)+4 \sqrt{2} \omega|\gamma|\left(E\left(u_{0}\right)\right)^{1 / 2}\right)^{1 / 2}}, \quad \text { if } 0<\gamma<\frac{3}{2}, \\
\frac{-2 \arctan \frac{\left(\frac{\gamma^{2}}{2} E\left(u_{0}\right)+4 \sqrt{2} \omega|\gamma|\left(E\left(u_{0}\right)\right)^{\frac{1}{2}}\right)^{1 / 2}}{m(0)},}{\left(\frac{\gamma^{2}}{2} E\left(u_{0}\right)+4 \sqrt{2} \omega|\gamma|\left(E\left(u_{0}\right)\right)^{1 / 2}\right)^{1 / 2}}, \quad \text { if } \frac{3}{2} \leq \gamma \leq 3, \\
\frac{-2 \arctan \frac{\left(\frac{(2 \gamma-3) \gamma}{2} E\left(u_{0}\right)+4 \sqrt{2} \omega|\gamma|\left(E\left(u_{0}\right)\right)^{1 / 2}\right)^{1 / 2}}{m(0)},}{\left(\frac{(2 \gamma-3) \gamma}{2} E\left(u_{0}\right)+4 \sqrt{2} \omega|\gamma|\left(E\left(u_{0}\right)\right)^{1 / 2}\right)^{1 / 2}}, \quad \text { if } \gamma>3 \quad \text { or } \gamma<0,
\end{array}\right.
$$

where $m(0):=\inf _{x \in \mathbb{R}}\left\{\gamma \partial_{x} u_{0}(x)\right\}$. The previous estimate is sharp.

\section{Acknowledgments}

This work was performed as a Visiting Researcher at Lund University. The author is very pleased to acknowledge the support and encouragement of Professor A Constantin as this work has developed. The author is also grateful to Professor J L Bona for his interest in these investigations and for helpful discussions. This work was partially supported by the National Natural Science Foundation of China and the Foundation of Zhongshan University Advanced Research Center. 


\section{References}

[1] Beals R, Sattinger D and Szmigielski J, Acoustic Scattering and the Extended Korteweg-de Vries Hierarchy, Adv. Math. 140 (1998), 190-206.

[2] Benjamin T B, Bona J L and Mahony J J, Model Equations for Long Waves in Nonlinear Dispersive Systems, Phil. Trans. R. Soc. (London) 272 (1972), 47-78.

[3] Camassa R and Holm D, An Integrable Shallow Water Equation with Peaked Solitons, Phys. Rev. Lett. 71 (1993), 1661-1664.

[4] Constantin A, The Hamiltonian Structure of the Camassa-Holm Equation, Exposition. Math. 15 (1997), 53-85.

[5] Constantin A, Global Existence of Solutions and Breaking Waves for a Shallow Water Equation: a Geometric Approach, Ann. Inst. Fourier (Grenoble) 50 (2000), 321-362.

[6] Constantin A, On the Scattering Problem for the Camassa-Holm Equation, Proc. R. Soc. London A457 (2001), 953-970.

[7] Constantin A and Escher J, Global Existence and Blow-up for a Shallow Water Equation, Ann. Sc. Norm. Sup. Pisa 26 (1998), 303-328.

[8] Constantin A and Escher J, Wave Breaking for Nonlinear Nonlocal Shallow Water Equations, Acta Mathematica 181 (1998), 229-243.

[9] Constantin A and Strauss W A, Stability of a Class of Solitary Waves in Compressible Elastic Rods, Phys. Lett. A270 (2000), 140-148.

[10] Dai H H, Model Equations for Nonlinear Dispersive Waves in a Compressible Mooney-Rivlin Rod, Acta Mech. 127 (1998), 193-207.

[11] Dai H H and Huo Y, Solitary Shock Waves and Other Travelling Waves in a General Compressible Hyperelastic Rod, Proc. R. Soc. London A456 (2000), 331-363.

[12] Danchin R, A Few Remarks on the Camassa-Holm Equation, Differential and Integral Equations 14 (2001), 953-988.

[13] Dodd R K, Eilbeck J C, Gibbon J D, and Morris H C, Solitons and Nonlinear Wave Equations, Academic Press, New York, 1984.

[14] Fokas A and Fuchssteiner B, Symplectic Structures, their Bäcklund Transformation and Hereditary Symmetries, Physica D4 (1981), 47-66.

[15] Himonas A A and Misiolek G, The Cauchy Problem for an Integrable Shallow Water Equation, Differential and Integral Equations 14 (2001), 821-831.

[16] Johnson R S, Camassa-Holm, Korteweg-de Vries and Related Models for Water Waves, J. Fluid. Mech. 457 (2002), 63-82.

[17] Kato T, Quasi-Linear Equations of Evolution, with Applications to Partial Differential Equations, in Spectral Theory and Differential Equations, Lecture Notes in Math., Vol. 448, Springer Verlag, Berlin, 1975, 25-70.

[18] Lenells J, The Scattering Approach for the Camassa-Holm Equation, J. Nonlinear Math. Phys. 9 (2002), 389-393. 
[19] Li Y and Olver P, Well-Posedness and Blow-up Solutions for an Integrable Nonlinearly Dispersive Model Wave Equation, J. Differential Equations 162 (2000), 27-63.

[20] Rodriguez-Blanco G, On the Cauchy Problem for the Camassa-Holm Equation, Nonlinear Anal. 46 (2001), 309-327. 